\title{
DISSIPAÇÃO DE ENDOSSULFAN EM AMOSTRAS DE DOIS TIPOS DE SOLOS BRASILEIROS TRATADAS EM LABORATÓRIO
}

TEREZINHA BONANHO PERES *

SOLANGE PAPINI **

MARA MARCHETTI ***

LUIZ CARLOS LUCHINI ${ }^{* * * *}$

\begin{abstract}
Avaliou-se a persistência e a degradação de Endossulfan em gleissolo húmico $(\mathrm{GH})$ e argissolo vermelho-amarelo (AA), com diferentes características químicas e físicas, tratados em laboratório com solução de ${ }^{14} \mathrm{C}$-endossulfan. Determinou-se a persistência a partir da produção de ${ }^{14} \mathrm{CO}_{2}$ aos 7, 14, 21 e 28 dias após o tratamento. A degradação foi avaliada aos 0, 30, 60 e 90 dias após o tratamento pela extração das amostras, análise dos extratos e combustão das amostras extraídas. $O$ radiocarbono presente (extraível e ligado) foi quantificado por espectrometria de cintilação em líquido. Alíquotas dos extratos foram analisadas por cromatografia em camada delgada (CCD) quanto à presença de produtos de degradação. Nos dois tipos de solo, a persistência ambiental e o padrão de degradação mostraram-e semelhantes. Os valores detectados para a produção de ${ }^{14} \mathrm{CO}_{2}$, em ambos os tipos de solo, evidenciaram meia-vida entre 90 e 180 dias. Ocorreu diminuição na recuperação de ${ }^{14} \mathrm{C}$-resíduos extraíveis e aumento de ${ }^{14} \mathrm{C}$-resíduos ligados aos 0, 30, 60 e 90 dias, sendo a porcentagem desses últimos maior no solo GH (cerca de $8 \%$ ) do que no solo AA (cerca de 6\%). A CCD revelou nos dois tipos de solos a presença dos produtos de degradação endossulfan-diol, logo após o tratamento, endossulfan-sulfato e outro nãoidentificado aos 30, 60 e 90 dias, provavelmente relacionados com a sua biodegradação.
\end{abstract}

PALAVRAS-CHAVE: INSETICIDA ORGANOCLORADO; ENDOSSULFAN - PERSISTENNCIA; BIODEGRADAÇÃO.

\footnotetext{
Pesquisador Científico III, Instituto Biológico, Centro de Proteção Ambiental (CPA), Laboratório de Ecologia de Agroquímicos (LEA), São Paulo, SP.

** Bióloga, D.Sc. em Ecologia, Instituto Biológico, CPA, LEA (e-mail: solangepapini@uol.com.br).

*** Engenheira Agrônoma, M.Sc. em Ciências, Instituto Biológico, CPA, LEA.

**** Pesquisador Científico VI, Instituto Biológico, CPA, LEA (luchini@biologico.sp.gov.br).
} 


\section{INTRODUÇÃO}

O inseticida organoclorado endossulfan [1,2,3,4,7,7-hexaclorobiciclo-(2,2,1)-2-hepteno-5,6-bisoximetileno] foi introduzido no mercado em 1956 pela Hoeschet AG (EUA). É utilizado em culturas de café, soja e algodão para o controle de diversas espécies de insetos e ácaros mediante contato e ingestão dos produtos formulados (BRASIL, 2000). O endossulfan técnico contém 90 a $95 \%$ da mistura de dois estéreo-isômeros $(\alpha$ e $\beta$ ), sendo o restante constituído por endossulfan-diol e endossulfan-éter cíclico (BROOKS, 1974). O composto apresenta elevada toxicidade aguda para mamíferos, estando enquadrado na classe toxicológica I. É pouco solúvel em solventes orgânicos e praticamente insolúvel em água (LARINI, 1999). Estável quando exposto à luz, apresenta baixa estabilidade em meio básico (WORTHING e HANCE, 1991). Tende a sofrer hidrólise lenta a endossulfan-diol e a endossulfandióxido sulfúrico (BROOKS, 1974; WORTHING e HANCE, 1977). Apresenta menor persistência relativa quando comparado com outros organoclorados, como DDT, lindano, heptacloro e outros (KAUR, KUMAR e DURAJA, 1997; GRAHAM-BRICE, 1981). Entretanto é considerado perigoso para o ambiente devido à sua persistência e alta toxicidade para muitos invertebrados como, por exemplo, minhocas e peixes (BRASIL, 2000).

O composto sofre biotransformação, tendo sido comprovada a ação de algumas espécies de algas em solos coreanos na produção dos metabólitos endossulfan-diol, endossulfan-hidroxiéter e endossulfan-lactona (LEE et al., 2003). Em solos brasileiros não existem informações sobre a ação da microbiota nesse processo.

Para minimizar os riscos da utilização do endossulfan na agricultura brasileira torna-se necessário ampliar o conhecimento de seu comportamento em solos tropicais. Neste trabalho estudou-se a degradação do inseticida endossulfan em amostras de dois tipos de solos brasileiros, tratadas em laboratório.

\section{MATERIAL E MÉTODOS}

\subsection{SOLO}

Foram utilizados dois solos, ou seja, Gleyssolo tiomórfico húmico $(\mathrm{GH})$ e Argissolo VermelhoAmarelo eutrófico (AA). Coletou-se a amostra do solo GH em campo de cultivo de bananeira do Instituto Biológico (São Paulo, SP) e do solo AA na usina de açúcar São João, em São João da Boa Vista (SP).

As amostras de solo foram retiradas da camada de 0 a $10 \mathrm{~cm}$ de profundidade do perfil, secas em temperatura ambiente e passadas em peneira de malha $2 \mathrm{~mm}$. Subamostras foram enviadas para análise no Laboratório de Solo e Nutrição de Plantas da Escola Superior de Agronomia "Luiz de Queiroz" (ESALQ/USP) e para o Instituto Campineiro de Análise de Solo e Adubo Ltda. (Campinas, $\mathrm{SP}$ ). No Laboratório de Ecologia de Agroquímicos do Instituto Biológico (SP) foram avaliadas as seguintes características biológicas de ambos os solos: ácido glutâmico para determinação da atividade microbiana ( $\mu$ g consumidas $\mathrm{g}^{-1}$ de solo dia-1), quantificação de fungos cultivados em meio de Martin e bactérias cultivadas em meio ágar nutritivo por meio da contagem de unidades formadoras de colônia (UFC g ${ }^{-1}$ ) de solo.

O solo $\mathrm{GH}$ apresentou pH 6,5, $88 \mathrm{~g} \mathrm{dm}^{-3}$ de matéria orgânica (MO), $200 \mathrm{~g} \mathrm{~kg}^{-1}$ de areia, $330 \mathrm{~g} \mathrm{~kg}^{-1}$ de silte e $470 \mathrm{~g} \mathrm{~kg}^{-1}$ de argila. Os valores bióticos encontrados foram: 0,19 $\mathrm{g} \mathrm{g} \mathrm{g}^{-1} \mathrm{dia}^{-1} \mathrm{de}$ ácido glutâmico; $1,710^{5} \mathrm{UFC} \mathrm{g}^{-1}$ de fungos e 1,6 107 $\mathrm{UFC} \mathrm{g}^{-1}$ de bactérias. Já o solo AA apresentou as seguintes características: $\mathrm{pH}$ 5,2; $15 \mathrm{~g} \mathrm{dm}^{-3}$ de MO; 657, 350 e $306 \mathrm{~g} \mathrm{~kg}^{-1}$ de areia, silte e argila, respectivamente. Suas avaliações biológicas resultaram em $0,13 \mu \mathrm{g} \cdot \mathrm{g}^{-1}$.dia-1 de ácido glutâmico; 1,0 $10^{5} \mathrm{UFC} \mathrm{g}^{-1}$ de fungos e $2,110^{7} \mathrm{UFC}^{-1}$ de bactérias. 
Subamostras de ambos os solos foram avaliadas no Laboratório de Ecologia de Agroquímicos do Instituto Biológico (SP) quanto a Capacidade Máxima de Retenção de Água (CMRA), conforme metodologia da EMBRAPA (FRIGHETTO e VALARINI, 2000). As amostras dos dois tipos de solo foram reumedecidas com água destilada a $70 \%$ da CMRA e incubadas por uma semana em estufa (BOD $347 \mathrm{G}$ ) a $30^{\circ} \mathrm{C}$ para reativação da microbiota do solo.

\subsection{INSETICIDA ENDOSSULFAN}

Foram utilizados endossulfan grau técnico, pureza química de $95 \%$, e padrões de $\alpha$-endossulfan, $\beta$-endossulfan, endossulfan-diol e endossulfan-sulfato, pureza química de $99 \%$, todos obtidos da Hoescht do Brasil SA. Obteve-se $0{ }^{14} \mathrm{C}$-endossulfan, com atividade específica de $6,8 \mathrm{GBq} \mathrm{mg}^{-1}$ e pureza radioquímica de 95\%, do Institute of Isotopes of Hungarian Academy of Sciences (Izinta, Hungria).

As soluções de ${ }^{14} \mathrm{C}$-endossulfan e de endossulfan grau técnico, utilizadas nos estudos, bem como dos produtos de degradação padrão foram preparadas em acetona.

\subsection{METODOLOGIA}

\subsubsection{Estudo de Persistência}

Determinou-se a persistência do endossulfan nos solos GH e AA a partir da quantificação de ${ }^{14} \mathrm{CO}_{2}$ produzido em 28 dias (BRASIL, 1997). Dois lotes de $50 \mathrm{~g}$ de cada tipo de solo (GH e AA) foram tratados com solução de ${ }^{14} \mathrm{C}$-endossulfan e endossulfan grau técnico. Um lote de cada tipo de solo (I-GH e I-AA) recebeu tratamento de modo que a concentração final fosse de $0,87 \mu \mathrm{g}$ de ingrediente ativo (i.a.) e $0,07 \mathrm{kBq} \mathrm{g}^{-1}$ de solo. Tratou-se o outro lote de cada tipo de solo (II-GH e II-AA) de modo que a concentração final fosse de 6,98 $\mu \mathrm{g}$ de i.a. e $0,07 \mathrm{kBq} \mathrm{g}^{-1}$ de solo. As amostras foram homogeneizadas por 20 minutos e então transferidas para frascos de vidro com capacidade de $250 \mathrm{~mL}$ e tampa de rosca. Havia no interior desses, frascos de cintilação com $5,0 \mathrm{~mL}$ de solução aquosa de hidróxido de potássio $0,2 \mathrm{~mol} \mathrm{~L}^{-1}$ para captura do dióxido de carbono produzido a partir da amostra de solo tratada. Monitorou-se a produção de ${ }^{14} \mathrm{CO}_{2}$ por meio de coleta de $\mathrm{KOH}$ e quantificação de triplicatas de $1,0 \mathrm{~mL}$ dessa solução aos $7,14,21$ e 28 dias por espectrometria de cintilação em líquido (L'ANNUNZIATA, 1979).

\subsubsection{Estudo da Degradação}

Amostras com $200 \mathrm{~g}$ dos solos GH e AA foram tratadas com solução de ${ }^{14} \mathrm{C}$-endossulfan e endossulfan grau técnico, obtendo-se concentração final de $0,74 \mu \mathrm{g}$ de i.a. $\mathrm{g}^{-1}$ de solo e $0,78 \mathrm{kBq} \mathrm{g}^{-1} \mathrm{de}$ solo. A concentração utilizada enquadrou-se na faixa indicada para a prática agrícola $\left(0,8910^{-6} \mathrm{~kg}\right.$ ha $^{-1}$ ). Após homogeneização, as amostras foram separadas em 4 grupos (em triplicatas) com $10 \mathrm{~g}$ de solo. Analisou-se o grupo 1 logo após o tratamento, sendo os demais grupos mantidos a $30^{\circ} \mathrm{C} \mathrm{e}$ analisados 30 (grupo 2), 60 (grupo 3) e 90 (grupo 4) dias após o tratamento.

Ao término dos tempos de incubação, as amostras dos solos de cada grupo foram submetidas à extração com $50 \mathrm{~mL}$ da mistura de hexano e acetona (8:2, v/v) por agitação mecânica durante 4 horas. Após a extração, as amostras ficaram em repouso até que houvesse decantação e o extrato pudesse ser separado do solo. Alíquotas de $1,0 \mathrm{~mL}$ (em triplicatas) dos extratos foram analisadas por espectrometria de cintilação em líquido (ECL) para quantificação da radioatividade, determinando-se a porcentagem de ${ }^{14} \mathrm{C}$-resíduos extraíveis. 
As amostras extraídas secaram em temperatura ambiente, submetendo-se as subamostras de $500 \mathrm{mg}$ (em triplicatas) à combustão em equipamento Biological Oxidazer. Após oxidação de todo radiocarbono remanescente $\mathrm{a}^{14} \mathrm{CO}_{2}$, os ${ }^{14} \mathrm{C}$-resíduos presentes no solo $\left({ }^{14} \mathrm{C}\right.$-resíduos ligados) foram quantificados.

\subsubsection{Análise Estatística}

Efetuou-se a análise estatística dos resultados usando o teste não-paramétrico de MannWhiteney, com nível de significância de 5\% (SIEGEL, 1975). Foram comparadas as porcentagens de produção de ${ }^{14} \mathrm{CO}_{2}$ nas amostras dos solos $\mathrm{GH}$ e AA, tratadas com baixa e alta concentração do inseticida. Também foram analisadas estatisticamente as porcentagens detectadas como ${ }^{14} \mathrm{C}$-resíduos extraíveis e ${ }^{14} \mathrm{C}$-resíduos ligados nas amostras de ambos os solos aos 0, 30, 60 e 90 dias após 0 tratamento inicial.

\subsubsection{Análise Cromatográfica}

O restante dos extratos foi concentrado à secura em rotaevaporador $a 40^{\circ} \mathrm{C}$ e ressuspendido em $1,0 \mathrm{~mL}$ de acetona. Determinou-se a presença do ${ }^{14} \mathrm{C}$-endossulfan ou de seus ${ }^{14} \mathrm{C}$-produtos de degradação por meio de cromatografia em camada delgada (CCD) em placas de sílica gel-60 $\mathrm{F}_{254}$, utilizando-se a mistura de solventes hexano:clorofórmio:acetona (9:3:1, v/v) como sistema de desenvolvimento. As cromatoplacas foram deixadas para secar até evaporação do sistema de solventes, dividindo-se cada uma em faixas de $1,0 \mathrm{~cm}$. A sílica foi raspada e colocada em frascos, contendo $10 \mathrm{~mL}$ de solução cintiladora para solvente orgânico (BRAY, 1960), para análise da radioatividade presente e quantificação dos ${ }^{14} \mathrm{C}$-produtos de degradação por ECL.

Calculou-se a recuperação do radiocarbono nos extratos e o proveniente da combustão do solo extraído como porcentagem do ${ }^{14} \mathrm{C}$-endossulfan inicialmente aplicado.

\section{RESULTADOS E DISCUSSÃO}

O estudo de persistência revelou produção de ${ }^{14} \mathrm{CO}_{2}$ em 28 dias de aproximadamente 3,5\% nas amostras do lote I-GH, tratadas com baixa concentração do inseticida $\left(0,87 \mu \mathrm{g} \mathrm{g}^{-1}\right)$. Quando tratadas com dose mais elevada $\left(6,98 \mu \mathrm{g} \mathrm{g}^{-1}\right.$, lote II-GH) a produção foi de apenas $1,5 \%$, embora a diferença não seja significativa $(T=15,5, p>0,06)$. Nas amostras de solo $A A$, tanto no lote I-AA como no lote II-AA, os valores encontrados ficaram em torno de $0,5 \%$ (Figura 1). Esses resultados indicam meia-vida para o endossulfan nos dois tipos de solo estudados entre 90 e 180 dias, podendo ser classificado como composto de média persistência ambiental (BRASIL, 1997). Também evidenciaram que no solo $\mathrm{AA}$, o teor de areia mais elevado associado à menor quantidade de matéria orgânica promoveu mineralização do ${ }^{14} \mathrm{C}$-endossulfan mais lenta, independentemente da concentração utilizada (embora as diferenças detectadas não tenham sido significativas $p>0,06$ ). Mais estudos devem ser realizados pois, segundo GRAHAM-BRYCE (1981), menor teor de matéria orgânica no solo pode estar relacionado com menor biodiversidade microbiana e comprometer a biodegradação do inseticida.

A extração do ${ }^{14} \mathrm{C}$-endossulfan, logo após o tratamento em ambos os solos, apresentou $100 \%$ de recuperação do composto, indicando que o sistema de solventes utilizado e a metodologia aplicada foram eficazes (Figura 2). Após 30, 60 e 90 dias do tratamento inicial, a porcentagem de ${ }^{14} \mathrm{C}$-extraíveis diminuiu em ambos os solos, sendo que as diferenças encontradas nas amostras dos solos GH e AA não foram significativas ( $T=19,0, p>0,06)$ (Figura 2). 
FIGURA 1 - PORCENTAGEM DE PRODUÇÃO DE ${ }^{14} \mathrm{CO}_{2}$ A PARTIR DO TRATAMENTO DE AMOSTRAS DE SOLO GH E AA COM BAIXA $\left(0,87 \mu \mathrm{g}\right.$ I.A. $\mathrm{g}^{-1}$ DE SOLO, LOTE I-GH E LOTE I-AA) E ALTA CONCENTRAÇÃO $\left(6,98 \mu \mathrm{g} \mathrm{I.A.} \mathrm{g}^{-1}\right.$ DE SOLO, LOTE II-GH E LOTE II-AA) DO INSETICIDA ENDOSSULFAN

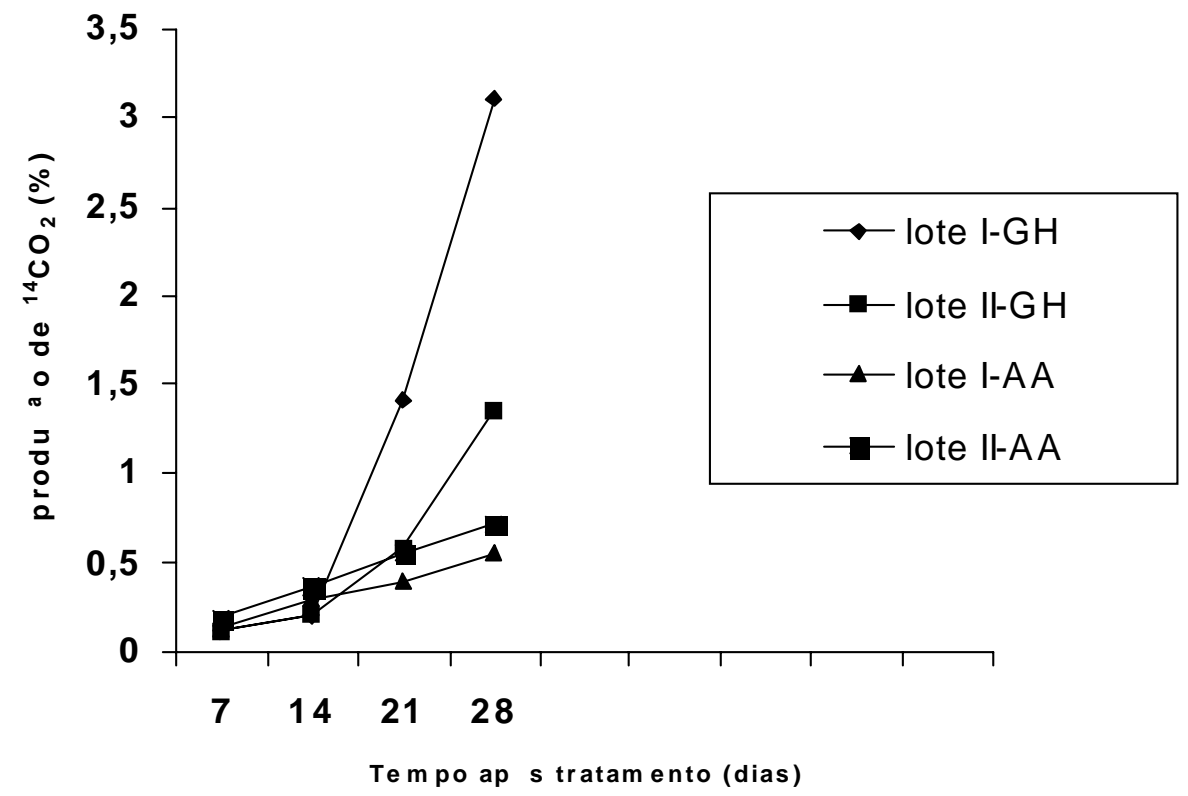

FIGURA 2 - DISTRIBUIÇÃO DO RADIOCARBONO RECUPERADO COMO ${ }^{14} \mathrm{C}$-RESÍDUOS EXTRAÍVEIS E ${ }^{14} \mathrm{C}$-RESÍDUOS LIGADOS PROVENIENTES DE AMOSTRAS DE SOLOS GH E AA TRATADAS COM ${ }^{14} \mathrm{C}$-ENDOSSULFAN

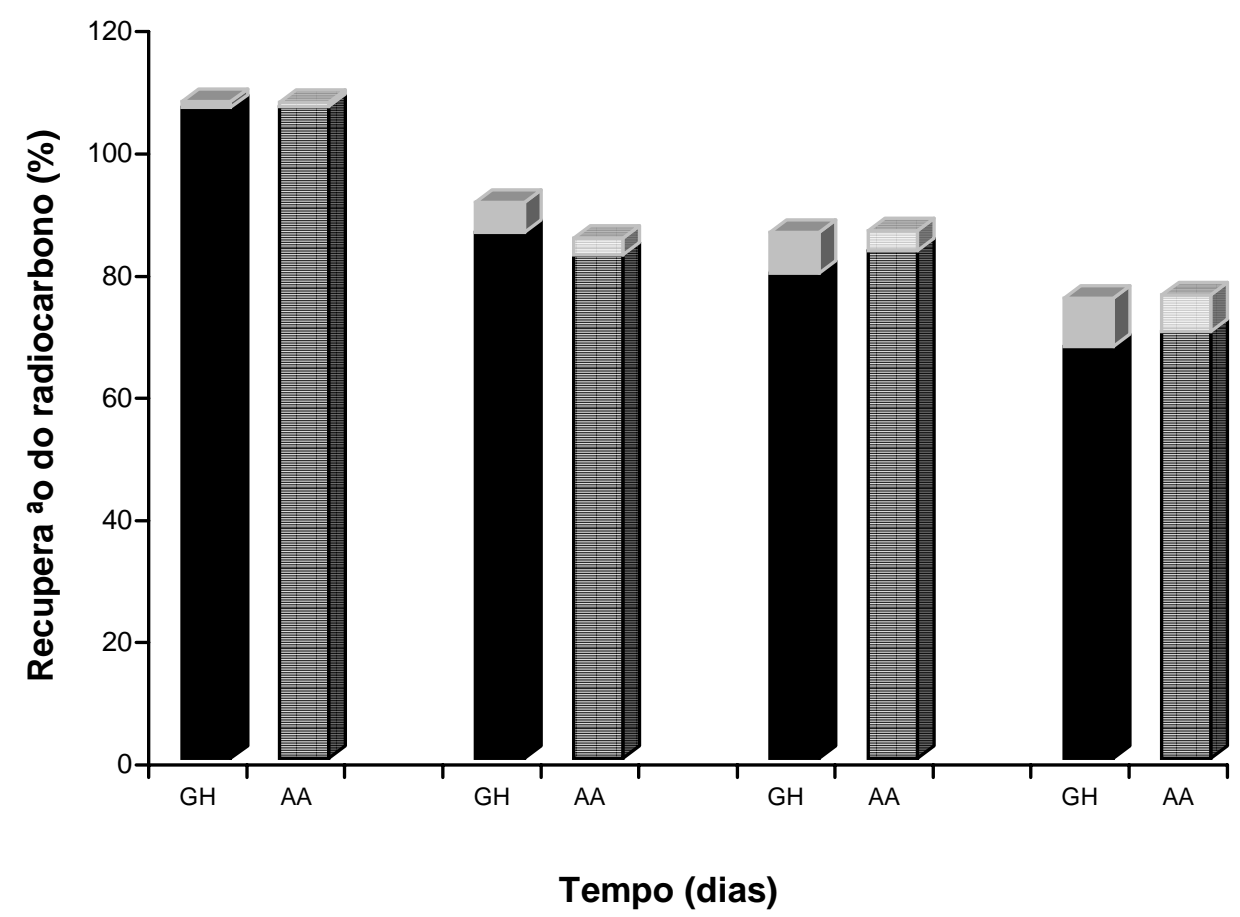

${ }^{14} \mathrm{C}$-resíduos extraíveis. ${ }^{14} \mathrm{C}$-resíduos ligados. 
Nas amostras de ambos os solos, após 90 dias foram recuperados cerca de $75 \%$ de ${ }^{14} \mathrm{C}$ resíduos de endossulfan (Figura 2). A diminuição da recuperação de ${ }^{14} \mathrm{C}$-resíduos sob a forma extraível correspondeu ao aumento dos ${ }^{14} \mathrm{C}$-resíduos ligados em ambos os solos. Foram detectados cerca de $8 \%$ e $6 \%$ de ${ }^{14} \mathrm{C}$-resíduos ligados nas amostras dos solos GH e AA, respectivamente (Figura 2). Embora as diferenças encontradas nas amostras de ambos os tipos de solos em todos os tempos estudados (30, 60 e 90 dias após a aplicação de ${ }^{14} \mathrm{C}$-endossulfan) não sejam significativas ( $T=13,0$, $\mathrm{p}>0,06$ ), as porcentagens de ${ }^{14} \mathrm{C}$-resíduos ligados foram maiores no solo $\mathrm{GH}$ (Figura 2). Esse fato parece indicar que maior teor de matéria orgânica no solo GH favoreceu a retenção da molécula do inseticida, dificultando sua extração (SHALINI-SINGH et al., 1999). Entretanto, a confirmação dessa possibilidade exige mais estudos.

O fator de retenção $(\mathrm{Rf})$ dos isômeros que compõem o inseticida determinado a partir da CCD do ${ }^{14} \mathrm{C}$-endossulfan padrão foi de 0,60 para o isômero $\alpha$ e de 0,43 para o isômero $\beta$. A análise cromatográfica dos extratos obtidos logo após o tratamento dos solos revelou que em ambos, cerca de $35 \%$ dos resíduos correspondiam ao isômero $\alpha$ e cerca de $59 \%$ ao isômero $\beta$. Observou-se nesses extratos a presença de aproximadamente $4 \%$ do metabólito endossulfan-diol (Rf 0,10$)$, provavelmente já presente no composto técnico (Tabelas 1 e 2 ).

\section{TABELA 1 - PORCENTAGEM DE DISTRIBUIÇÃO DE RADIOCARBONO NOS EXTRATOS DO SOLO GH POR CROMATOGRAFIA EM CAMADA DELGADA IMEDIATAMENTE APÓS O TRATAMENTO E AOS 30, 60 E 90 DIAS}

\begin{tabular}{|c|c|c|c|c|c|c|}
\hline \multirow{2}{*}{$\begin{array}{c}\text { Tempo } \\
\text { (dias) }\end{array}$} & \multicolumn{3}{|c|}{ Endossulfan } & \multicolumn{3}{|c|}{ Metab litos } \\
\hline & $\begin{array}{c}\alpha \\
\mathrm{Rf}=0,60\end{array}$ & $\begin{array}{c}\beta \\
\mathrm{Rf}=0,43\end{array}$ & & $\begin{array}{c}\text { sulfato } \\
\mathrm{Rf}=0,35\end{array}$ & $\begin{array}{c}\text { diol } \\
\mathrm{Rf}=0,10\end{array}$ & $\begin{array}{c}\mathrm{n}^{\mathrm{a}} \text { o-identificado } \\
\mathrm{Rf}=0,23\end{array}$ \\
\hline 0 & 34,7 & 59,1 & 93,8 & 0 & 3,6 & 0 \\
\hline 30 & 10,9 & 46,1 & 57,0 & 40,8 & 0,8 & 0,5 \\
\hline 60 & 6,1 & 44,3 & 50,4 & 40,1 & 0,8 & 0,3 \\
\hline 90 & 4,8 & 32,3 & 37,1 & 60,5 & 1,1 & 0,6 \\
\hline
\end{tabular}

$\mathrm{Rf}=$ Fator de retenção

A degradação do isômero $\alpha$ foi maior do que a do isômero $\beta$, sendo verificadas porcentagens semelhantes em ambos os tipos de solo. A degradação do isômero $\beta$ foi mais acentuada no solo GH (Tabela 1).

Concomitantemente à diminuição da porcentagem dos isômeros do endossulfan houve o aparecimento de metabólitos. Foram detectados nos extratos obtidos a partir de 30 dias do tratamento dos solos outros dois metabólitos, além do endossulfan-diol. O endossulfan-sulfato com Rf 0,35 e outro metabólito não identificado com Rf 0,23 . No solo $\mathrm{GH}$, as porcentagens dos metabólitos endossulfansulfato e do não-identificado foram maiores do que as encontradas no solo AA (Tabelas 1 e 2). Provavelmente, 0 alto teor de matéria orgânica nesse tipo de solo favoreceu maior bioatividade sobre 0 composto (LEE et al., 2003; SUTHERLAND et al., 2002). O inverso ocorreu com as porcentagens de endossulfan-diol, as quais foram mais altas nos extratos do solo AA com pH menor que o solo $\mathrm{GH}$ (Tabela 2). Esse produto é comumente formado a partir de lenta degradação do endossulfan (BROOKS, 1974; WORTHING e HANCE, 1977), estando relacionado principalmente com maior teor de acidez do meio (METCALF, 1971). 


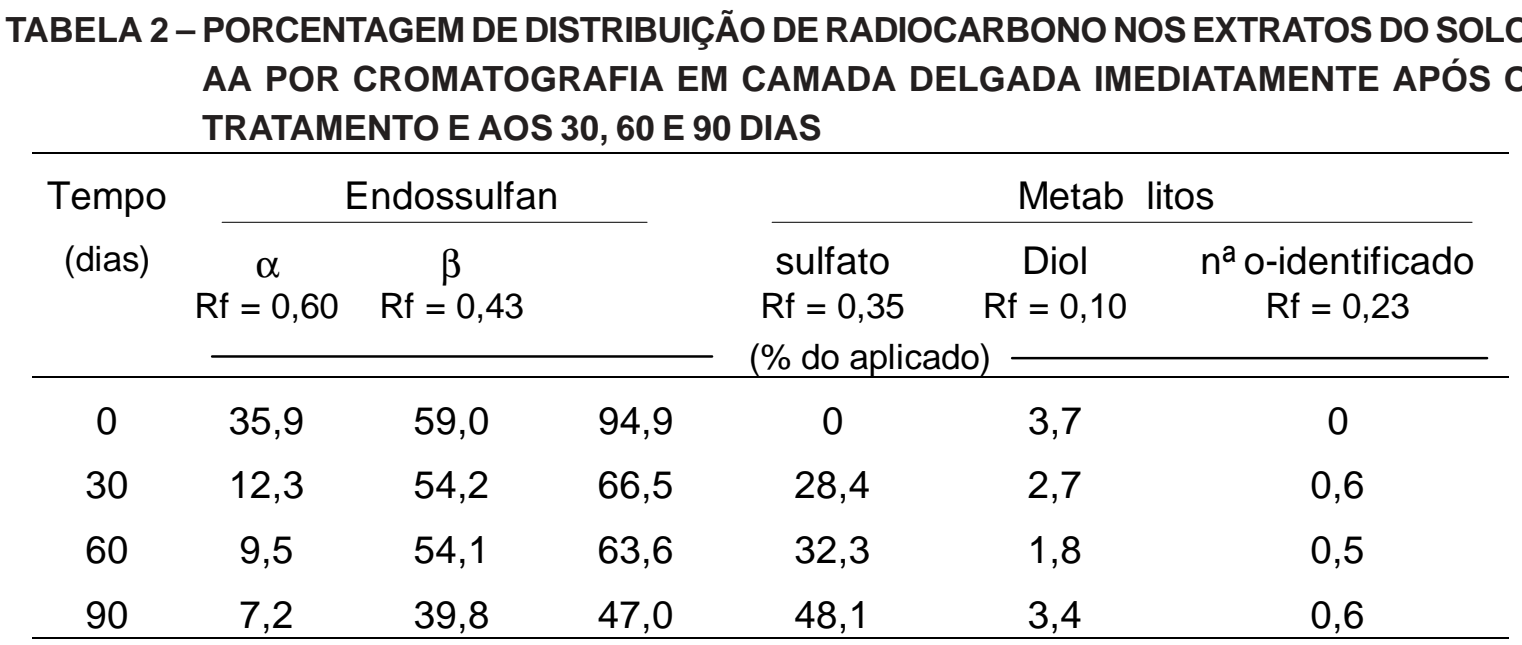

$\mathrm{Rf}=$ Fator de retenção.

\title{
4 CONCLUSÃO
}

A meia-vida do endossulfan nos dois tipos de solo estudados variou de 90 a 180 dias, sendo o produto classificado como de média persistência ambiental. O padrão de degradação do endossulfan mostrou-se semelhante nos dois tipos de solo. O produto de degradação endossulfan-diol parece resultar de degradação química, enquanto que o endossulfan-sulfato pode ser proveniente da atividade da microbiota edáfica.

\begin{abstract}
ENDOSULFAN DISSIPATION IN TWO BRAZILIAN SOIL SAMPLES TREATED IN LABORATORY

Persistant and degradation of Endosulfan in different soils (GH and $A A$ ) with distinct chemical and physical characteristics were evaluated by treatment with ${ }^{14} \mathrm{C}$-endosulfan solution. Persistance was determined from the production of ${ }^{14} \mathrm{CO}_{2}, 7$, 14, 21 and 28 days after treatment. Degradation was evaluated at $0,30,60$ and 90 days after treatment by sample extraction, extract analysis and combustion of extracted samples. The radiocarbon (extractable and bound) was quantified by cintilation spectroscopy. Aliquots of the extracts were analysed by thyn layer chromatography (TLC) for the presence of degradation products. In both soil samples, environmental persistance and degradation standard were similar. The values detected for the production of ${ }^{14} \mathrm{CO}_{2}$ in both soils evidenced half-life between 90 and 180 days. Reduction in the recuperation of ${ }^{14} \mathrm{C}$ - extractable residues occured and augmented for bound ${ }^{14} \mathrm{C}$ residues at $0,30,60$ and 90 days, being the latter percentage higher in GH soil (around 8\%) than in AA soil (around 6\%). TLC showed in both soils the presence of endosulfan-diol degradation products right after the treatment, endosulfan-sulphate and other non identified at 30,60 and 90 days, probably related with its biodegradation.
\end{abstract}

KEY-WORDS: ORGANOCHLORINE PESTICIDE; ENDOSULFAN - PERSISTAENCE; BIODEGRADATION.

\section{REFERÊNCIAS}

1 BRASIL. Ministério da Habitação, Urbanismo e Meio Ambiente. Secretaria Especial do Meio Ambiente. Secretaria de Tecnologia e Controle Ambiental. Coordenadoria de Toxicologia Ambiental. Manual de testes para avaliação da ecotoxicidade de agentes químicos. Brasília: SEMA, STC, 1997. Cap. E1.2 (Biodegradabilidade em solos).

2 BRASIL. Ministério da Saúde. Agência Nacional de Vigilância Sanitária. Disponível em: <http://www.anvisa.gov.br/ agrosia>. Acesso em: 30 set. 2000.

3 BRAY, G.A. A simple efficient liquid scintillator for counting aqueous solutions in a liquid scintillation counter. Analytical Biochemistry, v. 1, p. 279-285, 1960. 

184.

5 FRIGHETTO, R. T. S.; VALARINI, P. J. Indicadores biológicos e bioquímicos da qualidade do solo: manual técnico embrapa. Jaguariúna: EMBRAPA, 2000. p. 37-40.

6 GRAHAM-BRICE, I.G. The behaviour of pesticides in soil. In: GREENLAND, D.J.; HAYES, M.H.B. (Ed.). The chemistry of soil processes. [Washington]: John Wiley, 1981. p. 621-670.

7 KAUR, I.; KUMAR, A; DURAJA, P. Separation of endossulfan and its metabolites by GC and HPLC. Biomedical Chromatography, v. 11, n.1, p.33-35, 1997.

8 L'ANNUNZIATA, M.S. Liquid scintillation counting. In: RADIOTRACER in an agricultural chemistry. London: Academic Press, 1979. Cap. 4, p. 89-145.

9 LARINI, L. Toxicologia dos praguicidas. São Paulo: Manole, 1999.

10 LEE, S.E.; KIM, J.S.; KENNEDY, I.R.; PARK, J.W.; KWON, G.S.; KOH, S.C.; KIM, J.E. Biotransformation of an organochlorine inseticide, endosulfan, by Anabaena species. Journal of Agricultural Food Chemistry, v.51, n. 5, p. 1336-1340, 2003.

11 MESQUITA, R.B.; RÜEGG, E.F. Influência de agentes tensoativos na detecção da radiação beta. Ciência e Cultura, v.36, p. 446-450, 1984.

12 METCALF, R.L. The chemistry and biology of pesticides. In: WHITE-STEVENS, R. (Ed.). Pesticides in the environment. New York: Marcel Dekker, 1971. v. 1, Part. 1, cap. 1.

13 SHALINI-SINGH, P.; DUREJA, P.; KUMAR, S.; JAIN, M. M. Persistence of alpha and beta isomers of endosulphan and endosulphan-sulphate in diverse soils of India as influenced by flooding. Journal Environmental Science Health B, v. 34, n. 6, p. 965-974, 1999.

14 SIEGEL, S. Estatística não-paramétrica. São Paulo: McGraw-Hill, 1975. p. 131-143.

15 SUTHERLAND, T.D.; WEIR, K.M.; LACEY, M.J.; HORNE, I.; RUSSELL, R.J.; OAKESHATT, J.G. Enrichment of a microbial culture capable of degrading endosulphate, the toxic metabolite of endossulfan. Jounal Applied Microbiology, v.92, n. 3, p. 541-548, 2002.

16 WORTHING, C. R.; HANCE, R. J. The pesticide manual. $5^{\text {th }}$ ed. London: British Crop Protection Council, 1977. p. 232.

17 WORTHING, C. R.; HANCE, R. J. The pesticide manual. 9 $9^{\text {th }}$ ed. London: British Crop Protection Council, 1991. p. 332. 